\title{
Chemical and Dynamical Evolution of IZw18
}

\author{
Simone Recchi \\ Institut für theoretische Physik und Astrophysik, Kiel University and \\ Max-Planck Institut für Astrophysik, Garching bei München, Germany
}

\begin{abstract}
We study the effect of different star formation regimes on the dynamical and chemical evolution of the Dwarf Galaxy IZw18. To do that, we make use of a 2-D hydrocode coupled with detailed chemical yields originating from SNeII, SNeIa and low- and intermediate-mass stars. The results of our simulations are that IZw18 is a pretty young galaxy (we do not need to invoke stars older than $300 \mathrm{Myr}$ in order to reproduce the chemical properties of this object) and the last burst of star formation occurred at most 15 Myr ago.
\end{abstract}

\section{Introduction}

IZw18 has been considered until recently as the best candidate for a truly "young" galaxy, but recent deep Color-Magnitude Diagrams (CMDs), both in the optical (Aloisi et al. 1999) and in the near infrared (Östlin 2000) revealed the presence of at least two stellar populations. This galaxy has been extensively studied by many authors and nowadays we know with reasonable accuracy the abundances in the $\mathrm{HII}$ and in the $\mathrm{H}$ I regions.

We simulate a model galaxy resembling IZw18 by means of a 2-D hydrocode, coupled with detailed chemical yields originating from SNeII, SNeIa and winds from Intermediate-Mass Stars (IMS). The thermalization efficiency (the fraction of the initial explosion energy which is available to thermalize the ISM) for SNeII is assumed to be low, since they explode in a medium, on average, cold and dense. SNeIa, instead, explode with some delay and the medium is already heated and diluted by the previous activity of SNeII, thus we can assume a higher thermalization efficiency.

\section{Results}

\subsection{Bursting mode of star formation}

We consider either a single, instantaneous burst of Star Formation (SF), or two instantaneous bursts separated by a quiescent period of 300 Myr. A Galactic Wind (GW) develops as a consequence of the energy injected during the starburst, carrying away mostly the freshly produced metals. However, due to the different explosion time-scales and thermalization efficiencies, the GW is mostly triggered by SNeIa. From the chemical point of view, iron-peak elements are ejected more easily than $\alpha$-elements, thus the net effect is to enhance $[\alpha / \mathrm{Fe}]$ 
ratios inside the galaxy. This effect is very strong in single-burst models and is milder in double-burst ones. The evolution of $\mathrm{C}, \mathrm{N}, \mathrm{O}$ in single and double burst models is shown in Fig. 1, left side (see Recchi et al. 2002 for more details about these results). The abundances are reproduced either for a single burst with an age of $\sim 31-35 \mathrm{Myr}$, or with a two-burst model with an age of 4-7 Myr. The first possibility is ruled out by the presence of old stars and by the (very blue) colors of IZw18, so the only acceptable solution is a very young burst superimposed on an older stellar population.
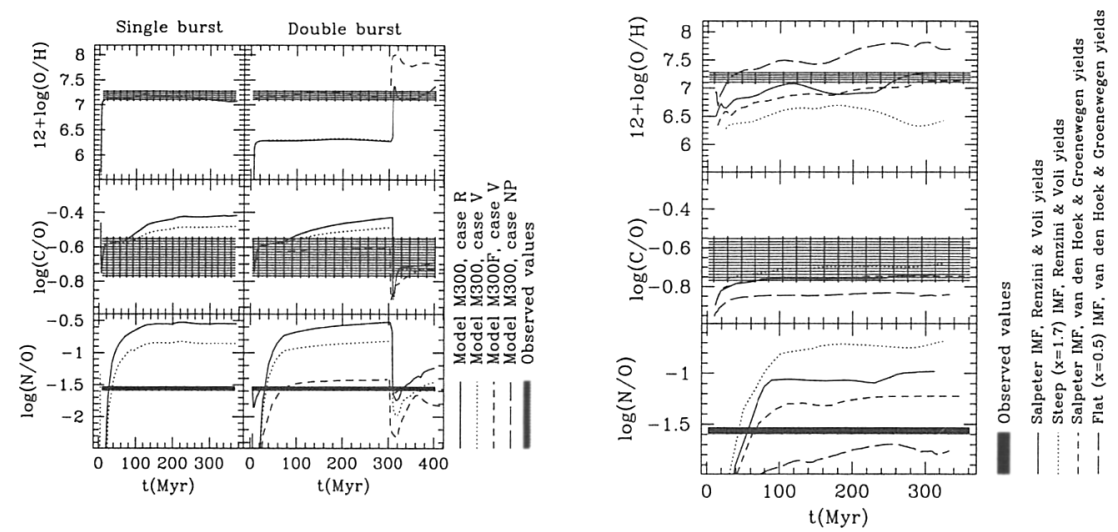

Figure 1. C, N, O evolution in bursting models (left figure) and in continuous burst model (right figure).

\subsection{Continuous burst models}

According to the results of Aloisi et al. (1999), we adopt a Star Formation constant for almost $300 \mathrm{Myr}$ (with SFR $=6 \cdot 10^{-3} \mathrm{M}_{\odot} \mathrm{y}^{-1}$ ), with superimposed a second, five times more vigorous burst, lasting for $5 \mathrm{Myr}$. The resulting evolution of $\mathrm{O}, \mathrm{C} / \mathrm{O}, \mathrm{N} / \mathrm{O}$ for different IMF slopes and different IMS yields is shown in Fig. 1, right side. These models cannot reproduce the observed N/O ratio. The $\mathrm{O}$ produced during the last burst of SF is in fact not enough to compensate the $\mathrm{N}$ coming from IMS. A better fit is obtained by making use of new yields by Meynet \& Maeder (2002), although they do not take into consideration the third dredge-up. The age of the last burst can be constrained by using the other elements, obtaining the best fit for a burst younger than 15 Myr.

Acknowledgments. SR acknowledges financial support from the Alexander von Humboldt Foundation and Deutsche Forschungsgemeinschaft (DFG).

\section{References}

Aloisi, A., Tosi, M., \& Greggio, L., 1999, AJ, 118, 302

Meynet, G., \& Maeder, A., 2002, A\&A, 390, 561

Östlin, G., 2000, ApJ, 535, L99

Recchi, S., Matteucci, F., D'Ercole, A., \& Tosi, M., 2002, A\&A, 384, 799 